\title{
Orlicz strongly convergent sequences with some applications to Fourier series and statistical convergence
}

Kuldip Raj ${ }^{1}$ and Adem Kilicman ${ }^{2}$

${ }^{1}$ Department of Mathematics Shri Mata Vaishno Devi University, Katra-182320, J \& K, India

${ }^{2}$ Department of Mathematics, University Putra Malaysia, 43400 UPM, Serdang, Selangor, Malaysia

Received: 13 January 2018, Accepted: 4 March 2018

Published online: 16 March 2018.

\begin{abstract}
In the present work we introduce and study some strongly convergent sequence spaces of Orlicz functions using infinite matrix over $n$-normed spaces. We study some algebraic and topological properties of these sequence spaces. A necessary and sufficient condition for strongly convergent sequences is obtained. Finally, we study some applications of these sequences for Fourier series and statistical convergence.
\end{abstract}

Keywords: Orlicz function, paranorm space, $\Lambda^{2}$-strongly convergent, statistical convergent, $n$-normed spaces.

\section{Introduction and preliminaries}

Let $l_{\infty}, c$ and $c_{0}$, respectively be the Banach spaces of bounded, convergent and null sequences $x=\left(x_{k}\right)$, normed by $\|x\|=\sup _{k}\left|x_{k}\right|$, where $k \in \mathbb{N}$, where $\mathbb{N}=\{0,1,2, \ldots\}$.

Mursaleen and Noman [20] introduced the notion of $\lambda$-convergent and $\lambda$-bounded sequences. Let $\lambda=\left\{\lambda_{k}\right\}_{k=0}^{\infty}$ be a strictly increasing sequence of positive real numbers tending to infinity. A sequence $x=\left(x_{k}\right) \in w$ is said to be $\lambda$-convergent to the number $L$ and called the $\lambda$-limit of $x$ if $\Lambda_{m}(x) \rightarrow L$ as $m \rightarrow \infty$, where

$$
\Lambda_{m}(x)=\frac{1}{\lambda_{m}} \sum_{k=0}^{m}\left(\lambda_{k}-\lambda_{k-1}\right) x_{k}
$$

A sequence $x=\left(x_{k}\right) \in w$ is $\lambda$-bounded if $\sup _{m}\left|\Lambda_{m}(x)\right|<\infty$. It is well known [20] that if $\lim _{m} x_{m}=a$ in the ordinary sense of convergence, then

$$
\lim _{m}\left(\frac{1}{\lambda_{m}}\left(\sum_{k=0}^{m}\left(\lambda_{k}-\lambda_{k-1}\right)\left|x_{k}-a\right|\right)\right)=0 .
$$

This implies that

$$
\lim _{m}\left|\Lambda_{m}(x)-a\right|=\lim _{m}\left|\frac{1}{\lambda_{m}} \sum_{k=0}^{m}\left(\lambda_{k}-\lambda_{k-1}\right)\left(x_{k}-a\right)\right|=0
$$

which gives that $\lim _{m} \Lambda_{m}(x)=a$ and we say $x=\left(x_{k}\right)$ is $\lambda$-convergent to $a$. Here and in the sequel, we shall use the convention that any term with a negative subscript is equal to zero, that is, $\lambda_{-1}=x_{-1}=0$. 
In [14] Kórus gave a new appropriate definition for the $\Lambda^{2}$-strong convergence by generalizing the original $\Lambda$-strong convergence concept given by Móricz [23]. Moreover, Kòrus [15] generalized the results on the $L^{1}$ convergence of Fourier series. Let $\Lambda=\left\{\lambda_{k}: k=0,1, \ldots\right\}$ be a non-decreasing sequence of positive numbers tending to $\infty$. A sequence $\left(x_{k}\right)$ of complex numbers converges $\Lambda^{2}$-strongly to a complex number $x$ if

$$
\lim _{n} \Lambda^{2}(x)-x=\lim _{n \rightarrow \infty} \frac{1}{\lambda_{n}} \sum_{k=0}^{n}\left|\lambda_{k}\left(x_{k}-x\right)-\lambda_{k-2}\left(x_{k-2}-x\right)\right|=0,
$$

with the argument $\lambda_{-1}=\lambda_{-2}=x_{-1}=x_{-2}=0$. In this paper we use the idea of $\Lambda^{2}$-strong convergence and we study these type of convergence over $n$-normed spaces.

In 1971 Lindenstrauss and Tzafriri [16] first investigated Orlicz sequence spaces in detail with certain aims in Banach space theory. An Orlicz function $M:[0, \infty) \rightarrow[0, \infty)$ is a continuous, non-decreasing and convex such that $M(0)=0$, $M(x)>0$ for $x>0$ and $M(x) \longrightarrow \infty$ as $x \longrightarrow \infty$. An Orlicz function $\mathscr{M}=\left(M_{k}\right)$ is said to satisfy $\Delta_{2}$-condition if there exist constants $a, K>0$ and a sequence $c=\left(c_{k}\right)_{k=1}^{\infty} \in l_{+}^{1}$ (the positive cone of $l^{1}$ ) such that the inequality

$$
M_{k}(2 u) \leq K M_{k}(u)+c_{k}
$$

holds for all $k \in \mathbb{N}$ and $u \in \mathbb{R}^{+}$, whenever $M_{k}(u) \leq a$.

Let $w$ be the set of all real or complex sequences. Lindenstrauss and Tzafriri [16] used the idea of Orlicz function to construct the sequence space,

$$
\ell_{M}=\left\{x=\left(x_{k}\right) \in w: \sum_{k=1}^{\infty} M\left(\frac{\left|x_{k}\right|}{\rho}\right)<\infty, \text { for some } \rho>0\right\}
$$

is known as an Orlicz sequence space. The space $\ell_{M}$ is a Banach space with the norm,

$$
\|x\|=\inf \left\{\rho>0: \sum_{k=1}^{\infty} M\left(\frac{\left|x_{k}\right|}{\rho}\right) \leq 1\right\} .
$$

Also it was shown in [16] that every Orlicz sequence space $\ell_{M}$ contains a subspace isomorphic to $\ell_{p}(p \geq 1)$. A sequence $\mathscr{M}=\left(M_{k}\right)$ of Orlicz functions is said to be Musielak-Orlicz function (see [17], [21]).

Let $X$ and $Y$ be two sequence spaces and $A=\left(a_{n k}\right)$ be an infinite matrix of real or complex numbers $a_{n k}$, where $n, k \in \mathbb{N}$. Then we say that $A$ defines a matrix mapping from $X$ into $Y$ if for every sequence $x=\left(x_{k}\right) \in X$, the sequence $A x=\left\{A_{n}(x)\right\}$ is in $Y$, where

$$
A_{n}(x)=\sum_{k} a_{n k} x_{k} \quad(n \in \mathbb{N})
$$

converges for each $n \in \mathbb{N}$. By $(X, Y)$ we denote the class of all matrices $A$ such that $A: X \rightarrow Y$.

For a sequence space $\mathrm{X}$, the matrix domain $X_{A}$ of an infinite matrix $A$ is defined by

$$
X_{A}=\left\{x=\left(x_{k}\right) \in w: A x \in X\right\}
$$

which is also a sequence space (see [30]).

A satisfactory theory of 2-normed spaces was initially developed by Gähler [11] in the mid of 1960's, while that of 
$n$-normed spaces one can see in Misiak [18]. Since then, this concept has been studied by many authors and obtained various results (see [12], [13]). Let $n \in \mathbb{N}$ and $X$ be a linear space over the field of real numbers $\mathbb{R}$ of dimension $d$, where $d \geq n \geq 2$. A real valued function $\|\cdot, \cdots, \cdot\|$ on $X^{n}$ satisfying the following four conditions:

(1) $\left\|x_{1}, x_{2}, \cdots, x_{n}\right\|=0$ if and only if $x_{1}, x_{2}, \cdots, x_{n}$ are linearly dependent in $X$,

(2) $\left\|x_{1}, x_{2}, \cdots, x_{n}\right\|$ is invariant under permutation,

(3) $\left\|\alpha x_{1}, x_{2}, \cdots, x_{n}\right\|=|\alpha||| x_{1}, x_{2}, \cdots, x_{n}||$ for any $\alpha \in \mathbb{R}$, and

(4) $\left\|x+x^{\prime}, x_{2}, \cdots, x_{n}\right\| \leq\left\|x, x_{2}, \cdots, x_{n}\right\|+\left\|x^{\prime}, x_{2}, \cdots, x_{n}\right\|$

is called an $n$-norm on $X$, and the pair $(X,\|\cdot, \cdots, \cdot\|)$ is called a $n$-normed space over the field $\mathbb{R}$.

For example, we may take $X=\mathbb{R}^{n}$ being equipped with the $n$-norm $\left\|x_{1}, x_{2}, \cdots, x_{n}\right\|_{E}=$ the volume of the $n$-dimensional parallelopiped spanned by the vectors $x_{1}, x_{2}, \cdots, x_{n}$ which may be given explicitly by the formula

$$
\left\|x_{1}, x_{2}, \cdots, x_{n}\right\|_{E}=\left|\operatorname{det}\left(x_{i j}\right)\right|,
$$

where $x_{i}=\left(x_{i 1}, x_{i 2}, \cdots, x_{i n}\right) \in \mathbb{R}^{n}$ for each $i=1,2, \cdots, n$.

Let $(X,\|\cdot, \cdots, \cdot\|)$ be an $n$-normed space of dimension $d \geq n \geq 2$ and $\left\{a_{1}, a_{2}, \cdots, a_{n}\right\}$ be linearly independent set in $X$. Then the following function $\|\cdot, \cdots, \cdot\|_{\infty}$ on $X^{n-1}$ as defined by

$$
\left\|x_{1}, x_{2}, \cdots, x_{n-1}\right\|_{\infty}=\max \left\{\left\|x_{1}, x_{2}, \cdots, x_{n-1}, a_{i}\right\|: i=1,2, \cdots, n\right\}
$$

is called an $(n-1)$-norm on $X$ with respect to $\left\{a_{1}, a_{2}, \cdots, a_{n}\right\}$.

A sequence $\left(x_{k}\right)$ in a $n$-normed space $(X,\|\cdot, \cdots, \cdot\|)$ is said to converges to some $L \in X$ if

$$
\lim _{k \rightarrow \infty}\left\|x_{k}-L, z_{1}, \cdots, z_{n-1}\right\|=0 \text { for every } z_{1}, \cdots, z_{n-1} \in X .
$$

A sequence $\left(x_{k}\right)$ in a $n$-normed space $(X,\|\cdot, \cdots, \cdot\|)$ is said to be Cauchy if

$$
\lim _{k, p \rightarrow \infty}\left\|x_{k}-x_{p}, z_{1}, \cdots, z_{n-1}\right\|=0 \text { for every } z_{1}, \cdots, z_{n-1} \in X .
$$

If every Cauchy sequence in $X$ converges to some $L \in X$, then $X$ is said to be complete with respect to the $n$-norm. Any complete $n$-normed space is said to be $n$-Banach space.

Let $X$ be a linear metric space. A function $p: X \rightarrow \mathbb{R}$ is called paranorm, if

(1) $p(x) \geq 0$ for all $x \in X$,

(2) $p(-x)=p(x)$ for all $x \in X$,

(3) $p(x+y) \leq p(x)+p(y)$ for all $x, y \in X$,

(4) if $\left(\lambda_{n}\right)$ is a sequence of scalars with $\lambda_{n} \rightarrow \lambda$ as $n \rightarrow \infty$ and $\left(x_{n}\right)$ is a sequence of vectors with $p\left(x_{n}-x\right) \rightarrow 0$ as $n \rightarrow \infty$, then $p\left(\lambda_{n} x_{n}-\lambda x\right) \rightarrow 0 ;$ as; $n \rightarrow \infty$.

A paranorm $p$ for which $p(x)=0$ implies $x=0$ is called total paranorm and the pair $(X, p)$ is called a total paranormed space. It is well known that the metric of any linear metric space is given by some total paranorm (see [33], Theorem 10.4.2, P-183). For more details about this type of sequence spaces (see [1], [4], [22], [24], [25], [26], [27], [28], [32]) and [5] - [19] references therein. 
A sequence $\left(x_{k}\right)$ of complex numbers is said to $\Lambda^{2}$-strongly convergent to a complex number $x$ with respect to a sequence of Orlicz functions and an infinite matrix if

$$
\lim _{n \rightarrow \infty} \frac{1}{\lambda_{n}} \sum_{k=0}^{n} a_{n k}\left[u_{k} M_{k}\left(\left\|\frac{\lambda_{k}\left(x_{k}-x\right)-\lambda_{k-2}\left(x_{k-2}-x\right)}{\rho}, z_{1}, \ldots, z_{n-1}\right\|\right)\right]^{p_{k}}=0 \text { for some } \rho>0
$$

with the agreement $\lambda_{-1}=\lambda_{-2}=x_{-1}=x_{-2}=0$. Let $\mathscr{M}=\left(M_{k}\right)$ be a sequence of Orlicz functions, $p=\left(p_{k}\right)$ be a bounded sequence of positive real numbers and $u=\left(u_{k}\right)$ be a sequence of strictly positive real numbers, $A=\left(a_{n k}\right)$ be an infinite matrix and $(X,\|\cdot, \cdots, \cdot\|)$ is an $n$-normed space. Let $\Lambda=\left\{\lambda_{k}\right\}$ be a non-decreasing sequence of positive numbers tending to $\infty$. In the present paper we define the following classes of sequences:

$$
\begin{gathered}
{\left[\Lambda^{2}, \mathscr{M}, A, u, p,\|., \ldots, .\|\right]=\left\{x=\left(x_{k}\right): \frac{1}{\lambda_{n}} \sum_{k=0}^{n} a_{n k}\left[u_{k} M_{k}\left(\left\|\frac{\lambda_{k}\left(x_{k}-x\right)-\lambda_{k-2}\left(x_{k-2}-x\right)}{\rho}, z_{1}, \ldots, z_{n-1}\right\|\right)\right]^{p_{k}}=0 ; \text { as; } n \rightarrow \infty\right\}} \\
{\left[\Lambda^{2}, \mathscr{M}, A, u, p,\|., \ldots, .\|\right]_{0}=\left\{x=\left(x_{k}\right): \frac{1}{\lambda_{n}} \sum_{k=0}^{n} a_{n k}\left[u_{k} M_{k}\left(\left\|\frac{\lambda_{k} x_{k}-\lambda_{k-2} x_{k-2}}{\rho}, z_{1}, \ldots, z_{n-1}\right\|\right)\right]^{p_{k}}=0 \text { as } n \rightarrow \infty\right\}}
\end{gathered}
$$

and

$$
\left[\Lambda^{2}, \mathscr{M}, A, u, p,\|., \ldots, .\|\right]_{\infty}=\left\{x=\left(x_{k}\right): \sup _{n} \frac{1}{\lambda_{n}} \sum_{k=0}^{n} a_{n k}\left[u_{k} M_{k}\left(\left\|\frac{\lambda_{k} x_{k}-\lambda_{k-2} x_{k-2}}{\rho}, z_{1}, \ldots, z_{n-1}\right\|\right)\right]^{p_{k}}<\infty\right\} .
$$

Let us consider a few special cases of the above classes of sequences:

If $M(x)=x$, then the sequences $\left[\Lambda^{2}, \mathscr{M}, A, u, p,\|., \ldots,\|.\right],\left[\Lambda^{2}, \mathscr{M}, A, u, p,\|., \ldots, .\|\right]_{0}$ and $\left[\Lambda^{2}, \mathscr{M}\right.$, $A, u, p,\|., \ldots,\|.]_{\infty}$ reduces to $\left[\Lambda^{2}, A, u, p,\|., \ldots,\|.\right],\left[\Lambda^{2}, A, u, p,\|., \ldots, .\|\right]_{0}$ and $\left[\Lambda^{2}, A, u, p,\|., \ldots, .\|\right]_{\infty}$ as follows:

$$
\begin{gathered}
{\left[\Lambda^{2}, A, u, p,\|., \ldots, .\|\right]=\left\{x=\left(x_{k}\right): \frac{1}{\lambda_{n}} \sum_{k=0}^{n} a_{n k}\left[u_{k}\left(\left\|\frac{\lambda_{k}\left(x_{k}-x\right)-\lambda_{k-2}\left(x_{k-2}-x\right)}{\rho}, z_{1}, \ldots, z_{n-1}\right\|\right)\right]^{p_{k}}=0 \text { as } n \rightarrow \infty\right\}} \\
{\left[\Lambda^{2}, A, u, p,\|., \ldots, .\|\right]_{0}=\left\{x=\left(x_{k}\right): \frac{1}{\lambda_{n}} \sum_{k=0}^{n} a_{n k}\left[u_{k}\left(\left\|\frac{\lambda_{k} x_{k}-\lambda_{k-2} x_{k-2}}{\rho}, z_{1}, \ldots, z_{n-1}\right\|\right)\right]^{p_{k}}=0 \text { as } n \rightarrow \infty\right\}}
\end{gathered}
$$

and

$$
\left[\Lambda^{2}, A, u, p,\|., \ldots, .\|\right]_{\infty}=\left\{x=\left(x_{k}\right): \sup _{n} \frac{1}{\lambda_{n}} \sum_{k=0}^{n} a_{n k}\left[u_{k}\left(\left\|\frac{\lambda_{k} x_{k}-\lambda_{k-2} x_{k-2}}{\rho}, z_{1}, \ldots, z_{n-1}\right\|\right)\right]^{p_{k}}<\infty\right\} .
$$

If $p_{k}=1$ for all $k \in \mathbb{N}$, we shall write above sequences as

$$
\begin{gathered}
{\left[\Lambda^{2}, \mathscr{M}, A, u,\|., \ldots, .\|\right]=\left\{x=\left(x_{k}\right): \frac{1}{\lambda_{n}} \sum_{k=0}^{n} a_{n k}\left[u_{k} M_{k}\left(\left\|\frac{\lambda_{k}\left(x_{k}-x\right)-\lambda_{k-2}\left(x_{k-2}-x\right)}{\rho}, z_{1}, \ldots, z_{n-1}\right\|\right)\right]=0 \text { as } n \rightarrow \infty\right\}} \\
{\left[\Lambda^{2}, \mathscr{M}, A, u,\|., \ldots, .\|\right]_{0}=\left\{x=\left(x_{k}\right): \frac{1}{\lambda_{n}} \sum_{k=0}^{n} a_{n k}\left[u_{k} M_{k}\left(\left\|\frac{\lambda_{k} x_{k}-\lambda_{k-2} x_{k-2}}{\rho}, z_{1}, \ldots, z_{n-1}\right\|\right)\right]=0 \text { as } n \rightarrow \infty\right\}}
\end{gathered}
$$

and

$$
\left[\Lambda^{2}, \mathscr{M}, A, u,\|., \ldots, .\|\right]_{\infty}=\left\{x=\left(x_{k}\right): \sup _{n} \frac{1}{\lambda_{n}} \sum_{k=0}^{n} a_{n k}\left[u_{k} M_{k}\left(\left\|\frac{\lambda_{k} x_{k}-\lambda_{k-2} x_{k-2}}{\rho}, z_{1}, \ldots, z_{n-1}\right\|\right)\right]<\infty\right\}
$$


The following inequality will be used throughout the paper. If $0<h=\inf _{k} p_{k} \leq p_{k} \leq \sup _{k} p_{k}=H, K=\max \left\{1,2^{H-1}\right\}$, then

$$
\left|a_{k}+b_{k}\right|^{p_{k}} \leq \quad K\left\{\left|a_{k}\right|^{p_{k}}+\left|b_{k}\right|^{p_{k}}\right\}
$$

for all $k \in \mathbb{N}$ and $a_{k}, b_{k} \in \mathbb{C}$. Also $|a|^{p_{k}} \leq \max \left\{1,|a|^{H}\right\}$ for all $a \in \mathbb{C}$.

The main objective of this paper is to introduce the concept of strongly convergent sequence spaces of Orlicz functions using infinite matrix over $n$-normed spaces. We also make an effort to study some topological properties and prove some inclusion relations between these sequence spaces. Finally, by using the concept of strong convergence we study statistical convergence and results related to Fourier series.

\section{Main results}

Theorem 1. Let $\mathscr{M}=\left(M_{k}\right)$ be a sequence of Orlicz functions, $p=\left(p_{k}\right)$ be a bounded sequence of positive real numbers and $u=\left(u_{k}\right)$ be a sequence of strictly positive real numbers and $A=\left(a_{n k}\right)$ be an infinite matrix. Then the sequence spaces $\left[\Lambda^{2}, \mathscr{M}, A, u, p,\|., \ldots,\|.\right],\left[\Lambda^{2}, \mathscr{M}, A, u, p,\|., \ldots, .\|\right]_{0}$ and $\left[\Lambda^{2}, \mathscr{M}, A, u, p,\|., \ldots, .\|\right]_{\infty}$ are linear over the complex field $\mathbb{C}$.

Proof. Suppose $x=\left(x_{k}\right)$ and $y=\left(y_{k}\right) \in\left[\Lambda^{2}, \mathscr{M}, A, u, p,\|., \ldots, .\|\right]_{0}$. Then

$$
\frac{1}{\lambda_{n}} \sum_{k=0}^{n} a_{n k}\left[u_{k} M_{k}\left(\left\|\frac{\lambda_{k} x_{k}-\lambda_{k-2} x_{k-2}}{\rho_{1}}, z_{1}, \ldots, z_{n-1}\right\|\right)\right]^{p_{k}}=0 \text { as } n \rightarrow \infty \text { for some } \rho_{1}>0
$$

and

$$
\frac{1}{\lambda_{n}} \sum_{k=0}^{n} a_{n k}\left[u_{k} M_{k}\left(\left\|\frac{\lambda_{k} y_{k}-\lambda_{k-2} y_{k-2}}{\rho_{2}}, z_{1}, \ldots, z_{n-1}\right\|\right)\right]^{p_{k}}=0 \text { as } n \rightarrow \infty \text { for some } \rho_{2}>0 .
$$

Let $\rho_{3}=\max \left(2|\alpha| \rho_{1}, 2|\beta| \rho_{2}\right)$. Since $\mathscr{M}=\left(M_{k}\right)$ is a non-decreasing and convex so by using inequality (3), we have

$$
\begin{aligned}
& \frac{1}{\lambda} \sum_{k=0}^{n} a_{n k}\left[u_{k} M_{k}\left(\left\|\frac{\alpha\left(\lambda_{k} x_{k}-\lambda_{k-2} x_{k-2}\right)+\beta\left(\lambda_{k} y_{k}-\lambda_{k-2} y_{k-2}\right)}{\rho_{3}}, z_{1}, \ldots, z_{n-1}\right\|\right)\right]^{p_{k}} \\
& \leq K \frac{1}{\lambda_{n}} \sum_{k=0}^{n} \frac{1}{2^{p_{k}}} a_{n k}\left[u_{k} M_{k}\left(\left\|\frac{\alpha\left(\lambda_{k} x_{k}-\lambda_{k-2} x_{k-2}\right)}{\rho_{1}}, z_{1}, \ldots, z_{n-1}\right\|\right)\right]^{p_{k}} \\
& +K \frac{1}{\lambda_{n}} \sum_{k=0}^{n} \frac{1}{2^{p_{k}}} a_{n k}\left[u_{k} M_{k}\left(\left\|\frac{\beta\left(\lambda_{k} y_{k}-\lambda_{k-2} y_{k-2}\right)}{\rho_{2}}, z_{1}, \ldots, z_{n-1}\right\|\right)\right]^{p_{k}} \\
& \leq K \frac{1}{\lambda_{n}} \sum_{k=0}^{n} a_{n k}\left[u_{k} M_{k}\left(\left\|\frac{\alpha\left(\lambda_{k} x_{k}-\lambda_{k-2} x_{k-2}\right)}{\rho_{1}}, z_{1}, \ldots, z_{n-1}\right\|\right)\right]^{p_{k}} \\
& +K \frac{1}{\lambda_{n}} \sum_{k=0}^{n} a_{n k}\left[u_{k} M_{k}\left(\left\|\frac{\beta\left(\lambda_{k} y_{k}-\lambda_{k-2} y_{k-2}\right)}{\rho_{2}}, z_{1}, \ldots, z_{n-1}\right\|\right)\right]^{p_{k}} \\
& =0 \text { as } n \rightarrow \infty .
\end{aligned}
$$

Thus, $\alpha x+\beta y \in\left[\Lambda^{2}, \mathscr{M}, A, u, p\|., \ldots, .\|\right]_{0}$. This proves that $\left[\Lambda^{2}, \mathscr{M}, A, u, p\|., \ldots, .\|\right]_{0}$ is a linear space. Similarly, we can prove that $\left[\Lambda^{2}, \mathscr{M}, A, u, p,\|., \ldots,\|.\right]$ and $\left[\Lambda^{2}, \mathscr{M}, A, u, p,\|., \ldots, .\|\right]_{\infty}$ are linear spaces.

Theorem 2. Let $\mathscr{M}=\left(M_{k}\right)$ be a sequence of Orlicz functions, $p=\left(p_{k}\right)$ be a bounded sequence of positive real numbers and $u=\left(u_{k}\right)$ a sequence of strictly positive real numbers and $A=\left(a_{n k}\right)$ be an infinite matrix. Then 
$\left[\Lambda^{2}, \mathscr{M}, A, u, p,\|., \ldots, .\|\right]_{0}$ is a paranormed space with paranorm

$$
g(x)=\inf \left\{(\rho)^{\frac{p_{k}}{K}}: \frac{1}{\lambda_{n}} \sum_{k=0}^{n}\left[a_{n k}\left[u_{k} M_{k}\left(\left\|\frac{\lambda_{k} x_{k}-\lambda_{k-2} x_{k-2}}{\rho}, z_{1}, \ldots, z_{n-1}\right\|\right)\right]^{p_{k}}\right]^{\frac{1}{K}} \leq 1, \text { as } n \rightarrow \infty,\right\}
$$

where $H=\sup _{k} p_{k}<\infty$ and $K=\max \{1, H\}$.

Proof. (i) Clearly $g(x) \geq 0$ for $x=\left(x_{k}\right) \in\left[\Lambda^{2}, \mathscr{M}, A, u, p,\|, \ldots, .\|\right]_{0}$. By definition of Orlicz function, we get $g(0)=0$.

(ii) $g(-x)=g(x)$.

(iii) Let $x=\left(x_{k}\right), y=\left(y_{k}\right) \in\left[\Lambda^{2}, \mathscr{M}, A, u, p,\|., \ldots, .\|\right]_{0}$ there exist positive numbers $\rho_{1}$ and $\rho_{2}$ such that

$$
\frac{1}{\lambda_{n}} \sum_{k=0}^{n}\left[a_{n k}\left[u_{k} M_{k}\left(\left\|\frac{\lambda_{k} x_{k}-\lambda_{k-2} x_{k-2}}{\rho_{1}}, z_{1}, \ldots, z_{n-1}\right\|\right)\right]^{p_{k}}\right]^{\frac{1}{k}} \leq 1
$$

and

$$
\frac{1}{\lambda_{n}} \sum_{k=0}^{n}\left[a_{n k}\left[u_{k} M_{k}\left(\left\|\frac{\lambda_{k} x_{k}-\lambda_{k-2} x_{k-2}}{\rho_{2}}, z_{1}, \ldots, z_{n-1}\right\|\right)\right]^{p_{k}}\right]^{\frac{1}{K}} \leq 1 .
$$

Let $\rho=\rho_{1}+\rho_{2}$. Then by using Minkowski's inequality, we have

$$
\begin{aligned}
& \frac{1}{\lambda_{n}} \sum_{k=0}^{n} a_{n k}\left[u_{k} M_{k}\left(\left\|\frac{\left(\lambda_{k} x_{k}-\lambda_{k-2} x_{k-2}\right)+\left(\lambda_{k} y_{k}-\lambda_{k-2} y_{k-2}\right)}{\rho}, z_{1}, \ldots, z_{n-1}\right\|\right)\right]^{p_{k}} \\
& =\frac{1}{\lambda_{n}} \sum_{k=0}^{n} a_{n k}\left[u_{k} M_{k}\left(\left\|\frac{\left(\lambda_{k} x_{k}-\lambda_{k-2} x_{k-2}\right)+\left(\lambda_{k} y_{k}-\lambda_{k-2} y_{k-2}\right)}{\rho_{1}+\rho_{2}}, z_{1}, \ldots, z_{n-1}\right\|\right)\right]^{p_{k}} \\
& =\frac{1}{\lambda_{n}} \sum_{k=0}^{n} a_{n k}\left[u_{k} M_{k}\left(\left\|\frac{\lambda_{k} x_{k}-\lambda_{k-2} x_{k-2}}{\rho_{1}+\rho_{2}}, z_{1}, \ldots, z_{n-1}\right\|\right)\right]^{p_{k}} \\
& +\frac{1}{\lambda_{n}} \sum_{k=0}^{n} a_{n k}\left[u_{k} M_{k}\left(\left\|\frac{\lambda_{k} y_{k}-\lambda_{k-2} y_{k-2}}{\rho_{1}+\rho_{2}}, z_{1}, \ldots, z_{n-1}\right\|\right)\right]^{p_{k}} \\
& \leq\left(\frac{\rho_{1}}{\rho_{1}+\rho_{2}}\right) \frac{1}{\lambda_{n}} \sum_{k=0}^{n} a_{n k}\left[u_{k} M_{k}\left(\left\|\frac{\lambda_{k} x_{k}-\lambda_{k-2} x_{k-2}}{\rho_{1}}, z_{1}, \ldots, z_{n-1}\right\|\right)\right]^{p_{k}} \\
& +\left(\frac{\rho_{2}}{\rho_{1}+\rho_{2}}\right) \frac{1}{\lambda_{n}} \sum_{k=0}^{n} a_{n k}\left[u_{k} M_{k}\left(\left\|\frac{\lambda_{k} y_{k}-\lambda_{k-2} y_{k-2}}{\rho_{2}}, z_{1}, \ldots, z_{n-1}\right\|\right)\right]^{p_{k}} \\
& \leq 1 .
\end{aligned}
$$

and thus

$$
\begin{aligned}
g(x+y) & =\inf \left\{(\rho)^{\frac{p_{k}}{k}}: \frac{1}{\lambda_{n}} \sum_{k=0}^{n}\left[a_{n k}\left[u_{k} M_{k}\left(\left\|\frac{\left(\lambda_{k} x_{k}-\lambda_{k-2} x_{k-2}\right)+\left(\lambda_{k} y_{k}-\lambda_{k-2} y_{k-2}\right)}{\rho}, z_{1}, \ldots, z_{n-1}\right\|\right)\right]^{p_{k}}\right]^{\frac{1}{k}} \leq 1\right. \\
& \leq \inf \left\{\left(\rho_{1}\right)^{\frac{p_{k}}{K}}: \frac{1}{\lambda_{n}} \sum_{k=0}^{n}\left[a_{n k}\left[u_{k} M_{k}\left(\left\|\frac{\lambda_{k} x_{k}-\lambda_{k-2} x_{k-2}}{\rho_{1}}, z_{1}, \ldots, z_{n-1}\right\|\right)\right]^{p_{k}}\right]^{\frac{1}{K}} \leq 1\right\} \\
& +\inf \left\{\left(\rho_{2}\right)^{\frac{p_{k}}{K}}: \frac{1}{\lambda_{n}} \sum_{k=0}^{n}\left[a_{n k}\left[u_{k} M_{k}\left(\left\|\frac{\lambda_{k} y_{k}-\lambda_{k-2} y_{k-2}}{\rho_{2}}, z_{1}, \ldots, z_{n-1}\right\|\right)\right]^{p_{k}}\right]^{\frac{1}{K}} \leq 1\right\} .
\end{aligned}
$$

Therefore, $g(x+y)=g(x)+g(y)$. 
Finally, we prove that the scalar multiplication is continuous. Let $\lambda$ be any complex number. By definition,

$$
\begin{aligned}
g(\mu x) & =\inf \left\{(\rho)^{\frac{p_{k}}{K}}: \frac{1}{\lambda_{n}} \sum_{k=0}^{n}\left[a_{n k}\left[u_{k} M_{k}\left(\left\|\frac{\mu\left(\lambda_{k} x_{k}-\lambda_{k-2} x_{k-2}\right)}{\rho}, z_{1}, \ldots, z_{n-1}\right\|\right)\right]^{p_{k}}\right]^{\frac{1}{K}} \leq 1\right\} \\
& =\inf \left\{(|\mu| t)^{\frac{p_{k}}{K}}: \frac{1}{\lambda_{n}} \sum_{k=0}^{n}\left[a_{n k}\left[u_{k} M_{k}\left(\left\|\frac{\mu\left(\lambda_{k} x_{k}-\lambda_{k-2} x_{k-2}\right)}{\rho}, z_{1}, \ldots, z_{n-1}\right\|\right)\right]^{p_{k}}\right]^{\frac{1}{K}} \leq 1\right\},
\end{aligned}
$$

where $t=\frac{\rho}{|\mu|}>0$. Since $|\mu|^{p_{k}} \leq \max \left(1,|\mu|^{\text {sup } p_{k}}\right)$, we have

$$
g(\mu x) \leq \max \left(1,|\mu|^{\text {sup } p_{k}}\right) \inf \left\{t^{\frac{p_{k}}{K}}: \frac{1}{\lambda_{n}} \sum_{k=0}^{n}\left[a_{n k}\left[u_{k} M_{k}\left(\left\|\frac{\mu\left(\lambda_{k} x_{k}-\lambda_{k-2} x_{k-2}\right)}{t}, z_{1}, \ldots, z_{n-1}\right\|\right)\right]^{p_{k}}\right]^{\frac{1}{K}} \leq 1\right\} .
$$

So, the fact that the scalar multiplication is continuous follows from the above inequality. This completes the proof of the theorem.

Theorem 3. Suppose $\mathscr{M}=\left(M_{k}\right), \mathscr{M}^{\prime}=\left(M_{k}^{\prime}\right), \mathscr{M}^{\prime \prime}=\left(M_{k}^{\prime \prime}\right)$ are sequences of Orlicz functions, $p=\left(p_{k}\right)$ be a bounded sequence of positive real numbers, $u=\left(u_{k}\right)$ be a sequence of strictly positive real numbers, $A=\left(a_{n k}\right)$ be an infinite matrix and $0<h=\inf _{k} p_{k} \leq p_{k} \leq \sup _{k} p_{k}=H<\infty$. Then

(i) $\left[\Lambda^{2}, \mathscr{M}^{\prime}, A, u, p,\|., \ldots, .\|\right]_{0} \subseteq\left[\Lambda^{2}, \mathscr{M} \circ \mathscr{M}^{\prime}, A, u, p,\|, \ldots, .\|\right]_{0}$,

(ii) $\left[\Lambda^{2}, \mathscr{M}^{\prime}, A, u, p,\|., \ldots, .\|\right]_{0} \cap\left[\Lambda^{2}, \mathscr{M}^{\prime \prime}, A, u, p,\|., \ldots, .\|\right]_{0} \subseteq\left[\Lambda^{2}, \mathscr{M}^{\prime}+\mathscr{M}^{\prime \prime}, A, u, p,\|., \ldots, .\|\right]_{0}$.

Proof. (i) Let $x \in\left[\Lambda^{2}, \mathscr{M}^{\prime}, A, u, p,\|., \ldots, .\|\right]_{0}$. Then, we have

$$
\frac{1}{\lambda_{n}} \sum_{k=0}^{n} a_{n k}\left[u_{k} M_{k}\left(\left\|\frac{\lambda_{k} x_{k}-\lambda_{k-2} x_{k-2}}{\rho}, z_{1}, \ldots, z_{n-1}\right\|\right)\right]^{p_{k}}=0 .
$$

Let $\varepsilon>0$ and choose $\delta>0$ with $0<\delta<1$ such that $f_{k}(t)<\varepsilon$ for $0 \leq t \leq \delta$. We write $y_{k}=a_{n k}\left[u_{k} M_{k}^{\prime}\left(\left\|\frac{\lambda_{k} x_{k}-\lambda_{k-2} x_{k-2}}{\rho}, z_{1}, \ldots, z_{n-1}\right\|\right)\right]$ and let us consider

$$
\sum_{k=0}^{n}\left[M_{k}\left(y_{k}\right)\right]^{p_{k}}=\sum_{1}\left[M_{k}\left(y_{k}\right)\right]^{p_{k}}+\sum_{2}\left[M_{k}\left(y_{k}\right)\right]^{p_{k}}
$$

where the first summation is over $y_{k} \leq \delta$ and the second over $y_{k}>\delta$. Since $\mathscr{M}=\left(M_{k}\right)$ is continuous, we have

$$
\frac{1}{\lambda_{n}} \sum_{1}\left[M_{k}\left(y_{k}\right)\right]^{p_{k}}<
$$

and for $y_{k}>\delta$, we use the fact that

$$
y_{k}<\frac{y_{k}}{\delta} \leq 1+\frac{y_{k}}{\delta}
$$

By definition of Orlicz function, we have for $y_{k}>\delta$,

$$
M_{k}\left(y_{k}\right)<2 M_{k}(1) \frac{y_{k}}{\delta}
$$

Hence,

$$
\frac{1}{\lambda_{n}} \sum_{2}\left[M_{k}\left(y_{k}\right)\right]^{p_{k}} \leq \max \left(1,\left(2 M_{k}(1) \delta^{-1}\right)^{H}\right) \frac{1}{\lambda_{n}} \sum_{k=0}^{n}\left[y_{k}\right]^{p_{k}}
$$


So by equations (4) and (5), we have $\left[\Lambda^{2}, \mathscr{M}^{\prime}, A, u, p,\|., \ldots, .\|\right]_{0} \subseteq\left[\Lambda^{2}, \mathscr{M} \circ \mathscr{M}^{\prime}, A, u, p,\|., \ldots, .\|\right]_{0}$.

(ii) Let $x \in\left[\Lambda^{2}, \mathscr{M}^{\prime}, A, u, p,\|., \ldots, .\|\right]_{0} \cap\left[\Lambda^{2}, \mathscr{M}^{\prime \prime}, A, u, p,\|., \ldots, .\|\right]_{0}$. Then using inequality (3) it can be shown that

$$
x \in\left[\Lambda^{2}, \mathscr{M}^{\prime}+\mathscr{M}^{\prime \prime}, A, u, p,\|., \ldots, .\|\right]_{0}
$$

Hence, $\left[\Lambda^{2}, \mathscr{M}^{\prime}, A, u, p,\|., \ldots, .\|\right]_{0} \cap\left[\Lambda^{2}, \mathscr{M}^{\prime \prime}, A, u, p,\|., \ldots, .\|\right]_{0} \subseteq\left[\Lambda^{2}, \mathscr{M}^{\prime}+\mathscr{M}^{\prime \prime}, A, u, p,\|., \ldots, .\|\right]_{0}$.

Corollary 1. Suppose $\mathscr{M}=\left(M_{k}\right), \mathscr{M}^{\prime}=\left(M_{k}^{\prime}\right), M^{\prime \prime}=\left(\mathscr{M}_{k}^{\prime \prime}\right)$ are sequences of Orlicz functions, $p=\left(p_{k}\right)$ be a bounded sequence of positive real numbers and $u=\left(u_{k}\right)$ be a sequence of strictly positive real numbers. Then

(i) $\left[\Lambda^{2}, \mathscr{M}^{\prime}, A, u, p,\|., \ldots,\|.\right] \subseteq\left[\Lambda^{2}, \mathscr{M} \circ \mathscr{M}^{\prime}, A, u, p,\|., \ldots,\|.\right]$,

(ii) $\left[\Lambda^{2}, \mathscr{M}^{\prime}, A, u, p,\|., \ldots,\|.\right] \cap\left[\Lambda^{2}, \mathscr{M}^{\prime \prime}, A, u, p,\|., \ldots,\|.\right] \subseteq\left[\Lambda^{2}, \mathscr{M}^{\prime}+\mathscr{M}^{\prime \prime}, A, u, p,\|., \ldots,\|.\right]$,

(iii) $\left[\Lambda^{2}, \mathscr{M}^{\prime}, A, u, p,\|., \ldots, .\|\right]_{\infty} \subseteq\left[\Lambda^{2}, \mathscr{M} \circ \mathscr{M}^{\prime}, A, u, p,\|., \ldots, .\|\right]_{\infty}$,

(iv) $\left[\Lambda^{2}, \mathscr{M}^{\prime}, A, u, p,\|., \ldots, .\|\right]_{\infty} \cap\left[\Lambda^{2}, \mathscr{M}^{\prime \prime}, A, u, p,\|., \ldots, .\|\right]_{\infty} \subseteq\left[\Lambda^{2}, \mathscr{M}^{\prime}+\mathscr{M}^{\prime \prime}, A, u, p,\|., \ldots, .\|\right]_{\infty}$.

Proof. It is easy to prove by using Theorem (3), so we omit the details.

Theorem 4. Let $\mathscr{M}=\left(M_{k}\right)$ be a sequence of Orlicz functions. Then for any two sequences $p=\left(p_{k}\right)$ and $t=\left(t_{k}\right)$ of strictly positive real numbers, we have

(i) $\left[\Lambda^{2}, \mathscr{M}, A, u, p,\|., \ldots, .\|\right]_{0} \cap\left[\Lambda^{2}, \mathscr{M}, A, u, t,\|., \ldots, .\|\right]_{0} \neq \phi$,

(ii) $\left[\Lambda^{2}, \mathscr{M}, A, u, p,\|., \ldots,\|.\right] \cap\left[\Lambda^{2}, \mathscr{M}, A, u, t,\|., \ldots,\|.\right] \neq \phi$,

(iii) $\left[\Lambda^{2}, \mathscr{M}, A, u, p,\|., \ldots, .\|\right]_{\infty} \cap\left[\Lambda^{2}, \mathscr{M}, A, u, t,\|., \ldots, .\|\right]_{\infty} \neq \phi$.

Proof. (i) Since the zero element belongs to $\left[\Lambda^{2}, \mathscr{M}, A, u, p,\|., \ldots, .\|\right]_{0}$ and $\left[\Lambda^{2}, \mathscr{M}, A, u, t,\|., \ldots, .\|\right]_{0}$, thus the intersection is non-empty. Similarly, we can prove (ii) and (iii).

Proposition 1. Let $\mathscr{M}=\left(M_{k}\right)$ be a sequence of Orlicz functions, $p=\left(p_{k}\right)$ be a bounded sequence of positive real numbers and $u=\left(u_{k}\right)$ be a sequence of strictly positive real numbers. Then, we have

(i) $\left[\Lambda^{2}, A, u, p,\|., \ldots, .\|\right]_{0} \subseteq\left[\Lambda^{2}, \mathscr{M}, A, u, p,\|., \ldots, .\|\right]_{0}$,

(ii) $\left[\Lambda^{2}, A, u, p,\|., \ldots,\|.\right] \subseteq\left[\Lambda^{2}, \mathscr{M}, A, u, p,\|., \ldots,\|.\right]$,

(iii) $\left[\Lambda^{2}, A, u, p,\|., \ldots, .\|\right]_{\infty} \subseteq\left[\Lambda^{2}, \mathscr{M}, A, u, p,\|., \ldots, .\|\right]_{\infty}$.

Proof. It is obvious so, we omit the details.

Theorem 5. Let $0<p_{k} \leq r_{k}$ and $\left(\frac{r_{k}}{p_{k}}\right)$ be bounded, then $\left[\Lambda^{2}, \mathscr{M}, A, u, r,\|., \ldots,\|.\right] \subseteq\left[\Lambda^{2}, \mathscr{M}, A, u, p,\|., \ldots,\|.\right]$.

Proof. Let $x \in\left[\Lambda^{2}, \mathscr{M}, A, u, r,\|., \ldots,\|.\right], t_{k}=a_{n k}\left[u_{k} M_{k}\left(\left\|\frac{\left(\lambda_{k}\left(x_{k}-x\right)-\lambda_{k-2}\left(x_{k-2}-x\right)\right)}{\rho}, z_{1}, \ldots, z_{n-1}\right\|\right)\right]^{r_{k}}$ and $\mu_{k}=\left(\frac{p_{k}}{r_{k}}\right)$ for all $k \in \mathbb{N}$ so that $0<\mu \leq \mu_{k} \leq 1$. Define the sequence $\left(v_{k}\right)$ and $\left(w_{k}\right)$ as follows:

For $t_{k} \geq 1$, let $v_{k}=t_{k}$ and $w_{k}=0$ and for $t_{k}<1$, let $v_{k}=0$ and $w_{k}=t_{k}$.

Then, clearly for all $k \in \mathbb{N}$, we have $t_{k}=v_{k}+w_{k}, t_{k}^{\mu_{k}}=v_{k}^{\mu_{k}}+w_{k}^{\mu_{k}}, v_{k}^{\mu_{k}} \leq v_{k} \leq t_{k}$ and $w_{k}^{\mu_{k}} \leq w_{k}^{\mu}$. Therefore,

$$
\frac{1}{\lambda_{n}} \sum_{k=0}^{n} t_{k}^{\mu_{k}} \leq \frac{1}{\lambda_{n}} \sum_{k=0}^{n} t_{k}+\left[\frac{1}{\lambda_{n}} \sum_{k=0}^{n} w_{k}\right]^{\mu}
$$

Hence, $x \in\left[\Lambda^{2}, \mathscr{M}, A, u, p,\|., \ldots,\|.\right]$. Thus, $\left[\Lambda^{2}, \mathscr{M}, A, u, r,\|., \ldots,\|.\right] \subseteq\left[\Lambda^{2}, \mathscr{M}, A, u, p,\|., \ldots,\|.\right]$. This completes the proof of the theorem. 


\subsection{Some characterizations of strongly convergent sequences}

Lemma 1. Let $\mathscr{M}=\left(M_{k}\right)$ be a sequence of Orlicz functions, $p=\left(p_{k}\right)$ be a bounded sequence of positive real numbers and $u=\left(u_{k}\right)$ be a sequence of strictly positive real numbers. Then a sequence $\left(x_{k}\right)$ of complex numbers converges strongly to a number $x$ if and only if

(i) $M\left(x_{k}\right)$ converges to $M(x)$ in the ordinary sense and

(ii) $\lim _{n \rightarrow \infty} \frac{1}{\lambda_{n}} \sum_{k=2}^{n} a_{n k}\left[u_{k} M_{k}\left(\lambda_{k-2}\left\|\frac{x_{k}-x_{k-2}}{\rho}, z_{1}, \ldots, z_{n-1}\right\|\right)\right]^{p_{k}}=0$.

Proof. The representation

$$
\lambda_{k}\left(x_{k}-x\right)-\lambda_{k-2}\left(x_{k-2}-x\right)=\left(\lambda_{k}-\lambda_{k-2}\right)\left(x_{k}-x\right)+\lambda_{k-2}\left(x_{k}-x_{k-2}\right)
$$

implies both

$$
\begin{aligned}
& \frac{1}{\lambda_{n}} \sum_{k=0}^{n} a_{n k}\left[u_{k} M_{k}\left(\left\|\frac{\lambda_{k}\left(x_{k}-x\right)-\lambda_{k-2}\left(x_{k-2}-x\right)}{\rho}, z_{1}, \ldots, z_{n-1}\right\|\right)\right]^{p_{k}} \\
& \leq \frac{1}{\lambda_{n}} \sum_{k=0}^{n} a_{n k}\left[u_{k} M_{k}\left(\lambda_{k}-\lambda_{k-2}\right)\left(\left\|\frac{x_{k}-x}{\rho}, z_{1}, \ldots, z_{n-1}\right\|\right)\right]^{p_{k}}+\frac{1}{\lambda_{n}} \sum_{k=2}^{n} a_{n k}\left[u_{k} M_{k}\left(\lambda_{k-2}\left\|\frac{x_{k}-x_{k-2}}{\rho}, z_{1}, \ldots, z_{n-1}\right\|\right)\right]^{p_{k}}
\end{aligned}
$$

and

$$
\begin{aligned}
& \frac{1}{\lambda_{n}} \sum_{k=2}^{n} a_{n k}\left[u_{k} M_{k}\left(\lambda_{k-2}\left\|\frac{x_{k}-x_{k-2}}{\rho}, z_{1}, \ldots, z_{n-1}\right\|\right)\right]^{p_{k}} \leq \frac{1}{\lambda_{n}} \sum_{k=0}^{n} a_{n k}\left[u_{k} M_{k}\left(\left\|\frac{\lambda_{k}\left(x_{k}-x\right)-\lambda_{k-2}\left(x_{k-2}-x\right)}{\rho}, z_{1}, \ldots, z_{n-1}\right\|\right)\right]^{p_{k}} \\
& +\frac{1}{\lambda_{n}} \sum_{k=0}^{n} a_{n k}\left[u_{k} M_{k}\left(\lambda_{k}-\lambda_{k-2}\right)\left(\left\|\frac{x_{k}-x}{\rho}, z_{1}, \ldots, z_{n-1}\right\|\right)\right]^{p_{k}} .
\end{aligned}
$$

By using these inequalities together with the fact that $M\left(x_{k}\right)$ converging to $M(x)$, we have

$$
\lim _{n \rightarrow \infty} \frac{1}{\lambda_{n}} \sum_{k=0}^{n} a_{n k}\left[u_{k} M_{k}\left(\lambda_{k}-\lambda_{k-2}\right)\left(\left\|\frac{x_{k}-x}{\rho}, z_{1}, \ldots, z_{n-1}\right\|\right)\right]^{p_{k}}=0 .
$$

Hence, we get the necessity and sufficiency of both (i) and (ii).

Lemma 2. Let $\mathscr{M}=\left(M_{k}\right)$ be a sequence of Orlicz functions, $p=\left(p_{k}\right)$ be a bounded sequence of positive real numbers and $u=\left(u_{k}\right)$ be a sequence of strictly positive real numbers. Then a sequence $\left(x_{k}\right)$ of complex numbers converges strongly to a number $x$ if and only if

(i) $\mathscr{M}\left(\sigma_{n}\right)=\frac{1}{\lambda_{n}} \sum_{0 \leqq k \leqq n 2 \mid n-k} a_{n k}\left[u_{k} M_{k}\left(\lambda_{k}-\lambda_{k-2}\right)\left(\left\|\frac{x_{k}}{\rho}, z_{1}, \ldots, z_{n-1}\right\|\right)\right]^{p_{k}}$ converges to $F(x)$ in the ordinary sense and

(ii) $\lim _{n \rightarrow \infty} \frac{1}{\lambda_{n}} \sum_{k=2}^{n} a_{n k}\left[u_{k} M_{k}\left(\lambda_{k-2}\left\|\frac{x_{k}-x_{k-2}}{\rho}, z_{1}, \ldots, z_{n-1}\right\|\right)\right]^{p_{k}}=0$. 
Proof. Clearly,

$$
\begin{aligned}
& M\left(x_{n}\right)-M\left(\sigma_{n}\right)=\frac{1}{\lambda_{n}} \sum_{\substack{0 \leqq k \leqq n \\
2 \mid n-k}} a_{n k}\left[u_{k} M_{k}\left(\lambda_{k}-\lambda_{k-2}\right)\left(\left\|\frac{\left(x_{n}-x_{k}\right)}{\rho}, z_{1}, \ldots, z_{n-1}\right\|\right)\right]^{p_{k}} \\
& =\frac{1}{\lambda_{n}} \sum_{\substack{0 \leqq k \leqq n \\
2 \mid n-k}}\left[\left(\lambda_{k}-\lambda_{k-2}\right)\right]^{p_{k}} \sum_{\substack{k+2 \leqq j \leqq n \\
2 \mid n-j}} a_{n k}\left[u_{k} M_{k}\left(\left\|\frac{x_{j}-x_{j-2}}{\rho}, z_{1}, \ldots, z_{n-1}\right\|\right)\right]^{p_{k}} \\
& =\frac{1}{\lambda_{n}} \sum_{\substack{2 \leqq j \leqq n \\
2 \mid n-j}} a_{n k}\left[u_{k} M_{k}\left(\left\|\frac{x_{j}-x_{j-2}}{\rho}, z_{1}, \ldots, z_{n-1}\right\|\right)\right]^{p_{k}} \sum_{\substack{0 \leqq k \leqq j-2 \\
2 \mid n-k}}\left[\left(\lambda_{k}-\lambda_{k-2}\right)\right]^{p_{k}} \\
& =\frac{1}{\lambda_{n}} \sum_{\substack{2 \leqq j \leqq n \\
2 \mid n-j}} a_{n k}\left[u_{k} M_{k}\left(\lambda_{j-2}\left\|\frac{x_{j}-x_{j-2}}{\rho}, z_{1}, \ldots, z_{n-1}\right\|\right)\right]^{p_{k}} \text {. }
\end{aligned}
$$

Hence,

$$
\lim _{n \rightarrow \infty} \sup \left|M\left(x_{n}\right)-M\left(\sigma_{n}\right)\right| \leqq \lim _{n \rightarrow \infty} \sup \frac{1}{\lambda_{n}} \sum_{k=2}^{n} a_{n k}\left[u_{k} M_{k}\left(\lambda_{k-2}\left\|\frac{x_{k}-x_{k-2}}{\rho}, z_{1}, \ldots, z_{n-1}\right\|\right)\right]^{p_{k}} .
$$

According to lemma 10, for the necessity part, it is easy to see that $\lim _{n} M\left(\sigma_{n}\right)=M(x)$ which comes from the above inequality, condition (ii) of this lemma and $\lim _{n} M\left(x_{n}\right)=M(x)$. For the sufficient part, we only need $\lim _{n} M\left(x_{n}\right)=M(x)$ which also comes from the above inequality, condition (ii) of this lemma and $\lim _{n} M\left(\sigma_{n}\right)=M(x)$.

\section{Statistical convergence}

The notions of statistical convergence and convergence in density for sequences has been in the literature under different guises, since the early part of the last century. Statistical convergence was recently investigated by Fast [8] and Schoenberg [31] independently. Later on it was investigated from the sequence space point of view and linked with summability theory by Fridy [9], Connor [3], Salat [29], Fridy and Orhan [10] and many others. The notion of statistical convergence depends on the density of subsets of $\mathbb{N}$. A subset $E$ of $\mathbb{N}$ is said to have density $\delta(E)$ if

$$
\delta(E)=\lim _{n \rightarrow \infty} \frac{1}{n} \sum_{k=1}^{n} \chi_{E}(k) \text { exists }
$$

where $\chi_{E}$ is the characteristics function of $E$.

A sequence $x=\left(x_{k}\right)$ is said to be statistically convergent to L if for every $\varepsilon>0$,

$$
\lim _{n \rightarrow \infty} \frac{1}{n}\left|\left\{k \leq n:\left|x_{k}-L\right| \geq \varepsilon\right\}\right|=0
$$

In this case, we write $S-\lim _{k} x_{k}=L$ or $x \rightarrow L(S)$. The set of all statistical convergent sequences is denoted by $S$.

Definition 1.A sequence $x=\left(x_{k}\right)$ is said to be $\left[\Lambda^{2}, A, u, p,\|., \ldots,\|.\right]$-statistically convergent to $x$ if for any $\varepsilon>0$,

$$
\lim _{n \rightarrow \infty} \frac{1}{\lambda_{n}}\left|\left\{k \leq n:\left\|\frac{\lambda_{k}\left(x_{k}-x\right)-\lambda_{k-2}\left(x_{k-2}-x\right)}{\rho}, z_{1}, \ldots, z_{n-1}\right\| \geq \varepsilon\right\}\right|=0,
$$

where the vertical bars indicate the number of elements in the closed set. In this case, we write $S-\lim _{k} \Lambda_{k}^{2}(x)=x$ and the set of all statistically convergent sequences is denoted by $S\left(\Lambda^{2}\right)$. 
Theorem 6. Consider $\mathscr{M}=\left(M_{k}\right)$ be a sequence of Orlicz functions, $p=\left(p_{k}\right)$ be a bounded sequence of positive real numbers and $u=\left(u_{k}\right)$ be a sequence of strictly positive real numbers and $\sup _{k} p_{k}=H<\infty$. Then $\left[\Lambda^{2}, \mathscr{M}, A, u, p,\|., \ldots,\|.\right] \subset\left(S\left(\Lambda^{2}\right)\right)$.

Proof. Let $\quad x \in\left[\Lambda^{2}, \mathscr{M}, A, u, p,\|., \ldots,\|.\right]$. Take $\varepsilon>0, \sum_{1}$ denote the sum over $k \leq n$ with $\left\|\frac{\lambda_{k}\left(x_{k}-x\right)-\lambda_{k-2}\left(x_{k-2}-x\right)}{\rho}, z_{1}, \ldots, z_{n-1}\right\| \geq \varepsilon$ and $\sum_{2}$ denote the sum over $k \leq n$ with $\left\|\frac{\lambda_{k}\left(x_{k}-x\right)-\lambda_{k-2}\left(x_{k-2}-x\right)}{\rho}, z_{1}, \ldots, z_{n-1}\right\|<\varepsilon$. Then for each $z_{1}, \cdots, z_{n-1} \in X$, we obtain

$$
\begin{aligned}
& \frac{1}{\lambda_{n}} \sum_{k=0}^{n} a_{n k}\left[u_{k} M_{k}\left(\left\|\frac{\lambda_{k}\left(x_{k}-x\right)-\lambda_{k-2}\left(x_{k-2}-x\right)}{\rho}, z_{1}, \ldots, z_{n-1}\right\|\right)\right]^{p_{k}} \\
& =\frac{1}{\lambda_{n}}\left(\sum_{1} a_{n k}\left[u_{k} M_{k}\left(\left\|\frac{\lambda_{k}\left(x_{k}-x\right)-\lambda_{k-2}\left(x_{k-2}-x\right)}{\rho}, z_{1}, \ldots, . z_{n-1}\right\|\right)\right]^{p_{k}}\right. \\
& \left.+\sum_{2} a_{n k}\left[u_{k} M_{k}\left(\left\|\frac{\lambda_{k}\left(x_{k}-x\right)-\lambda_{k-2}\left(x_{k-2}-x\right)}{\rho}, z_{1}, \ldots, . z_{n-1}\right\|\right)\right]^{p_{k}}\right) \\
& \geq \frac{1}{\lambda_{n}} \sum_{1} a_{n k}\left[u_{k} M_{k}\left(\left\|\frac{\lambda_{k}\left(x_{k}-x\right)-\lambda_{k-2}\left(x_{k-2}-x\right)}{\rho}, z_{1}, \ldots, . z_{n-1}\right\|\right)\right]^{p_{k}} \\
& \geq \frac{1}{\lambda_{n}} \sum_{1}\left\{a_{n k}\left[u_{k} M_{k}(\varepsilon)\right]^{p_{k}}\right\} \geq \frac{1}{\lambda_{n}} \sum_{1} \min \left(\left\{a_{n k}\left[u_{k} M_{k}(\varepsilon)\right]^{h}\right\},\left\{u_{k}\left[a_{n k} M_{k}(\varepsilon)\right]^{H}\right\}\right) \\
& =\frac{1}{\lambda_{n}}\left|\left\{k \leq n:\left\|\frac{\lambda_{k}\left(x_{k}-x\right)-\lambda_{k-2}\left(x_{k-2}-x\right)}{\rho}, z_{1}, \ldots, . z_{n-1}\right\| \geq \varepsilon\right\}\right| \\
& \min \left(\left\{a_{n k}\left[u_{k} M_{k}(\varepsilon)\right]^{h}\right\},\left\{a_{n k}\left[u_{k} M_{k}(\varepsilon)\right]^{H}\right\}\right) .
\end{aligned}
$$

Hence, $x \in\left(S\left(\Lambda^{2}\right)\right)$. This completes the proof of the theorem.

Theorem 7. Consider $\mathscr{M}=\left(M_{k}\right)$ be bounded sequence of Orlicz functions, $p=\left(p_{k}\right)$ be a bounded sequence of positive real numbers and $u=\left(u_{k}\right)$ be a sequence of strictly positive real numbers and $0<\inf _{k} p_{k} \leq p_{k} \leq \sup _{k} p_{k}=H<\infty$. Then $\left(S\left(\Lambda^{2}\right)\right) \subset\left[\Lambda^{2}, \mathscr{M}, A, u, p\right]$.

Proof. Suppose that $\mathscr{M}=\left(M_{k}\right)$ be bounded. For given $\varepsilon>0, \sum_{1}$ denote the sum over $k \leq n$ with $\left\|\frac{\lambda_{k}\left(x_{k}-x\right)-\lambda_{k-2}\left(x_{k-2}-x\right)}{\rho}, z_{1}, \ldots, z_{n-1}\right\| \geq \varepsilon$ and $\sum_{2}$ denote the sum over $k \leq n$ with $\left\|\frac{\lambda_{k}\left(x_{k}-x\right)-\lambda_{k-2}\left(x_{k-2}-x\right)}{\rho}, z_{1}, \ldots, z_{n-1}\right\|<\varepsilon$. Since $\mathscr{M}=\left(M_{k}\right)$ be bounded there exists an integer $D$ such that $M_{k}(x)<D$ for all $x \geq 0$. Then for each $z_{1}, \cdots, z_{n-1} \in X$.

$$
\begin{aligned}
& \frac{1}{\lambda_{n}} \sum_{k=0}^{n} a_{n k}\left[u_{k} M_{k}\left(\left\|\frac{\lambda_{k}\left(x_{k}-x\right)-\lambda_{k-2}\left(x_{k-2}-x\right)}{\rho}, z_{1}, \ldots, z_{n-1}\right\|\right)\right]^{p_{k}} \\
& \leq \frac{1}{\lambda_{n}}\left(\sum_{1} a_{n k}\left[u_{k} M_{k}\left(\left\|\frac{\lambda_{k}\left(x_{k}-x\right)-\lambda_{k-2}\left(x_{k-2}-x\right)}{\rho}, z_{1}, \ldots, z_{n-1}\right\|\right)\right]^{p_{k}}\right. \\
& \left.+\sum_{2} a_{n k}\left[u_{k} M_{k}\left(\left\|\frac{\lambda_{k}\left(x_{k}-x\right)-\lambda_{k-2}\left(x_{k-2}-x\right)}{\rho}, z_{1}, \ldots, z_{n-1}\right\|\right)\right]^{p_{k}}\right) \\
& \leq \frac{1}{\lambda_{n}} \sum_{1} \max \left(\left\{a_{n k}\left[u_{k} D^{h}\right]\right\},\left\{a_{n k}\left[u_{k} D^{H}\right]\right\}\right)+\frac{1}{\lambda_{n}} \sum_{2}\left\{a_{n k}\left[u_{k} M_{k}(\varepsilon)\right]^{p_{k}}\right\} \\
& \leq \max \left(\left\{a_{n k}\left[u_{k} D^{h}\right]\right\},\left\{a_{n k}\left[u_{k} D^{H}\right]\right\}\right) \frac{1}{\lambda_{n}}\left|\left\{k \leq n:\left\|\frac{\lambda_{k}\left(x_{k}-x\right)-\lambda_{k-2}\left(x_{k-2}-x\right)}{\rho}, z_{1}, \ldots, z_{n-1}\right\| \geq \varepsilon\right\}\right| \\
& +\max \left(\left\{a_{n k}\left[u_{k} M_{k}(\varepsilon)\right]^{h}\right\},\left\{a_{n k}\left[u_{k} M_{k}(\varepsilon)\right]^{H}\right\}\right) .
\end{aligned}
$$

Hence, $x \in\left[\Lambda^{2}, \mathscr{M}, A, u, p,\|., \ldots,\|.\right]$. This completes the proof of the theorem. 


\section{Results for Fourier series}

The space of all $2 \pi$ periodic complex-valued continuous functions is a Banach space endowed with the norm $\|f\|_{C}=$ $\max _{t}|f(t)|$ and it is denoted by $C$. Let

$$
\frac{1}{2} a_{0}(f)+\sum_{k=1}^{\infty}\left(a_{k}(f) \cos k t+b_{k}(f) \sin k t\right)
$$

be the Fourier series of $f \in C$ and denoted by $s_{k}(f)$ the $k$-th partial sum of the series (4.1). We shall denote by $U_{\mathscr{M}, u, p,\|., \ldots, . .\|}$, $A_{\mathscr{M}, u, p,\|, \ldots, . .\|}$ and $S\left(\Lambda_{\mathscr{M}, u, p,\|, \ldots, . .\|}^{2}\right)$ respectively the classes of functions $f \in C$ whose Fourier series converges uniformly, absolutely and strongly on $[0,2 \pi)$. Now, if $f \in S\left(\Lambda_{\mathscr{M}, u, p,\|, \ldots, . .\|}^{2}\right)$ and $z_{1}, \ldots, z_{n-1} \in X$, then

$$
\lim _{n}\left\|\left(\frac{1}{\lambda_{n}} \sum_{k=0}^{n} a_{n k}\left[u_{k} M_{k}\left|\left(\frac{\lambda_{k}\left(s_{k}(f)-f\right)-\lambda_{k-2}\left(s_{k-2}(f)-f\right)}{\rho}, z_{1}, \ldots, z_{n-1}\right)\right|\right)\right]^{p_{k}}\right\|_{C}=0
$$

where $\lambda_{-1}=\lambda_{-2}=x_{-1}=x_{-2}=0$.

The space $U_{\mathscr{M}, u, p,\|, \ldots, . .\|}$ is a Banach space with the norm

$$
\|f\|_{U_{\mathscr{M}, u, p,\|, \ldots, .,\|}}=\sup _{k}\left\|a_{n k}\left[u_{k} M_{k}\left(\frac{s_{k}(f)}{\rho}, z_{1}, \ldots, z_{n-1}\right)\right]^{p_{k}}\right\|_{C}
$$

and the space $A_{\mathscr{M}, u, p,\|, \ldots, . .\|}$ is also a Banach space with the norm

$$
\|f\|_{A_{\mathscr{M}, u, p,\|, \ldots, .,\|}}=\frac{1}{2} a_{n k}\left[u_{k} M_{k}\left(\left\|\frac{a_{0}(f)}{\rho}, z_{1}, \ldots, z_{n-1}\right\|\right)\right]^{p_{k}}+\sum_{k=1}^{\infty} u_{k}\left[a_{n k} f_{k}\left(\left\|\frac{a_{k}(f)+b_{k}(f)}{\rho}, z_{1}, \ldots, z_{n-1}\right\|\right)\right]^{p_{k}} .
$$

One can easily prove that $U_{\mathscr{M}, u, p,\|, \ldots, .,\|}$ and $A_{\mathscr{M}, u, p,\|, \ldots, .,\|}$ are Banach spaces. We shall give the proof only for $S\left(\Lambda_{\mathscr{M}, u, p,\|, \ldots, . .\|}^{2}\right)$ in the next Theorem. Now, we define the norm

$$
\left.\|f\|_{S\left(\Lambda_{\mathscr{M}, u, p,\|, \ldots, .,\|}^{2}\right)}=\sup _{n} \| \frac{1}{\lambda_{n}} \sum_{k=0}^{n} a_{n k}\left[u_{k} M_{k} \mid\left(\frac{\lambda_{k}\left(s_{k}(f)-f\right)-\lambda_{k-2}\left(s_{k-2}(f)-f\right)}{\rho}, z_{1}, \ldots, z_{n-1}\right)\right]^{p_{k}} \mid\right) \|_{C},
$$

which is finite for every $f \in S\left(\Lambda_{\mathscr{M}, u, p,\|, \ldots, .,\|}^{2}\right)$. By using triangle inequality, we have

$$
\|f\|_{S\left(\Lambda_{\mathscr{M}, u, p,\|, \ldots, .,\|}^{2}\right)} \leq\|f\|_{C}+\sup _{n}\left\|\frac{1}{\lambda_{n}} \sum_{k=0}^{n} a_{n k}\left[u_{k} M_{k} \mid\left(\frac{\lambda_{k}\left(s_{k}(f)-f\right)-\lambda_{k-2}\left(s_{k-2}(f)-f\right)}{\rho}, z_{1}, \ldots, z_{n-1}\right)\right]^{p_{k}}\right\| \|_{C}
$$

and this sup is due to equation 9. The norm inequalities corresponding to equation (3.6) in [23] are

$$
\|f\|_{U_{\mathscr{M}, u, p,\|, \ldots, .,\|}} \leq\|f\|_{S\left(\Lambda_{\mathscr{M}, u, p,\|, \ldots, .,\|}^{2}\right)} \leq 2\|f\|_{A_{\mathscr{M}, u, p,\|, \ldots, .,\|}}
$$

which implies that $A_{\mathscr{M}, u, p,\|, \ldots, .,\|} \subset S\left(\Lambda_{\mathscr{M}, u, p,\|, \ldots, . .\|}^{2}\right) \subset U_{\mathscr{M}, u, p,\|, \ldots, . .\|}$

Lemma 3. Let $\mathscr{M}=\left(M_{k}\right)$ be a sequence of Orlicz functions, $p=\left(p_{k}\right)$ be a bounded sequence of positive real numbers and $u=\left(u_{k}\right)$ be a sequence of strictly positive real numbers. Then $f \in S\left(\Lambda_{\mathscr{M}, u, p,\|, \ldots, .,\|}^{2}\right)$ if and only if

(i) $\lim _{k}\left\|s_{k}(f)-f\right\|_{C}=0$ and

(ii) $\lim _{k}\left\|\frac{1}{\lambda_{n}} \sum_{k=2}^{n} a_{n k}\left[u_{k} M_{k}\left(\left|\frac{\lambda_{k-2}\left(a_{k}(f) \cos k t+b_{k}(f) \sin k t\right)}{\rho}, z_{1}, \ldots, z_{n-1}\right|\right)\right]^{p_{k}}\right\|_{C}=0$. 
Proof. It follows from the Lemma 1, so we omit it.

Let us denote

$$
\sigma_{n}(f)=\left|\frac{1}{\lambda_{n}} \sum_{k=0}^{n} a_{n k}\left[u_{k} M_{k}\left(\left\|\frac{\left(\lambda_{k}-\lambda_{k-2}\right) s_{k}(f)}{\rho}, z_{1}, \ldots, z_{n-1}\right\|\right)\right]^{p_{k}}\right| \quad(n=0,1, \ldots)
$$

Lemma 4. Let $\mathscr{M}=\left(M_{k}\right)$ be a sequence of Orlicz functions, $p=\left(p_{k}\right)$ be a bounded sequence of positive real numbers and $u=\left(u_{k}\right)$ be a sequence of strictly positive real numbers. Then $f \in S\left(\Lambda_{\mathscr{M}, u, p,\|, \ldots, . .\|}^{2}\right)$ if and only if

(i) $\lim _{k}\left\|\sigma_{k}(f)-f\right\|_{C}=0$ and

(ii) $\lim _{k}\left\|\frac{1}{\lambda_{n}} \sum_{k=2}^{n} a_{n k}\left[u_{k} M_{k}\left(\left|\frac{\lambda_{k-2}\left(a_{k}(f) \cos k t+b_{k}(f) \sin k t\right)}{\rho}, z_{1}, \ldots, z_{n-1}\right|\right)\right]^{p_{k}}\right\|_{C}=0$.

Theorem 8. The set $S\left(\Lambda_{\mathscr{M}, u, p,\|, \ldots, . .\|}^{2}\right)$ endowed with norm

$$
\|f\|_{S\left(\Lambda_{\mathscr{M}, u, p,\|, \ldots, .,\|}^{2}\right)}=\sup _{n}\left\|\frac{1}{\lambda_{n}} \sum_{k=0}^{n} a_{n k}\left[u_{k} M_{k}\left(\left|\frac{\lambda_{k}\left(s_{k}(f)-f\right)-\lambda_{k-2}\left(s_{k-2}(f)-f\right)}{\rho}, z_{1}, \ldots, z_{n-1}\right|\right)\right]^{p_{k}}\right\|_{C}
$$

is a Banach space.

Proof. The only thing we have to prove is completeness. For this, let $\left\{s_{j}\right\}_{j \geq 1}$ be a Cauchy sequence in the norm

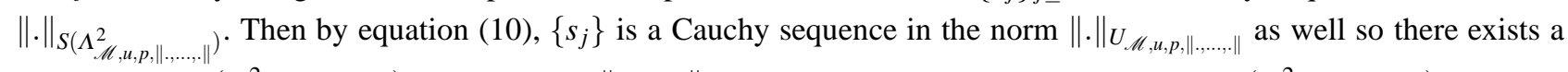
sequence $s \in S\left(\Lambda_{\mathscr{M}, u, p,\|, \ldots, .,\|}^{2}\right)$ such that $\lim _{j \rightarrow \infty}\left\|s_{j}-s\right\|_{U_{\mathscr{M}, u, p,\|, \ldots, .,\|}}=0$. Now, we show that $s \in S\left(\Lambda_{\mathscr{M}, u, p,\|, \ldots, . .\|}^{2}\right)$. Suppose $\varepsilon>0$, then by assumption there exists $v=v(\varepsilon)$ such that

$$
\left\|s_{j}-s_{i}\right\|_{S\left(\Lambda_{\mathscr{M}, u, p,\|, \ldots, .,\|}^{2}\right)} \leq \varepsilon \quad \text { for all } i, j \geq v
$$

Let $s_{j}=\left\{s_{j k}: k=0,1, \ldots\right\}$ and $s=\left\{s_{k}: k=0,1, \ldots\right\}$. We shall fix $i, n$. Similarly by equation (10), we have

$$
\frac{1}{\lambda_{n}} \sum_{k=0}^{n} a_{n k}\left[u_{k} M_{k}\left(\left\|\frac{\lambda_{k}\left(s_{j k}-s_{k}\right)-\lambda_{k-2}\left(s_{j(k-2)}-s_{k-2}\right)}{\rho}, z_{1}, \ldots, z_{2}\right\|\right)\right]^{p_{k}} \leq\left\|s_{j}-s\right\|_{U_{\mathscr{M}, u, p, \|, \ldots, .,}} \frac{1}{\lambda_{n}} \sum_{k=0}^{n}\left(\lambda_{k}+\lambda_{k-2}\right) \leq \varepsilon
$$

provided $j$ is large enough, due to $\lim _{j \rightarrow \infty}\left\|s_{j}-s\right\|_{U_{\mathscr{M}, u, p,\|, \ldots, .,\|}}=0$. Here $j$ depends on $n$ and $\varepsilon$ and assume that $j \geq v$. Applying triangle inequality by taking equations (12) and (13) into account we obtain that

$$
\begin{aligned}
& \frac{1}{\lambda_{n}} \sum_{k=0}^{n} a_{n k}\left[u_{k} M_{k}\left(\left\|\frac{\lambda_{k}\left(s_{j k}-s_{k}\right)-\lambda_{k-2}\left(s_{j(k-2)}-s_{k-2}\right)}{\rho}, z_{1}, \ldots, z_{n-1}\right\|\right)\right]^{p_{k}} \\
& \leq \frac{1}{\lambda_{n}} \sum_{k=0}^{n} a_{n k}\left[u_{k} M_{k}\left(\left\|\frac{\lambda_{k}\left(s_{j k}-s_{i k}\right)-\lambda_{k-2}\left(s_{j(k-2)}-s_{i(k-2)}\right)}{\rho}, z_{1}, \ldots, z_{n-1}\right\|\right)\right]^{p_{k}} \\
& +\frac{1}{\lambda_{n}} \sum_{k=0}^{n} a_{n k}\left[u_{k} M_{k}\left(\left\|\frac{\lambda_{k}\left(s_{i k}-s_{k}\right)-\lambda_{k-2}\left(s_{i(k-2)}-s_{k-2}\right)}{\rho}, z_{1}, \ldots, z_{n-1}\right\|\right)\right]^{p_{k}} \\
& \leq\left\|s_{j}-s_{i}\right\|_{S\left(\Lambda_{\mathscr{M}, u, p, \|, \ldots, .,}^{2}\right)}+\varepsilon=2 \varepsilon,
\end{aligned}
$$

for $j \geq v$. Since this hold for any $n \geq 0$, by definition $\left\|s_{j}-s_{i}\right\|_{S\left(\Lambda_{\mathscr{M}, u, p,\|, \ldots, .,\|}^{2}\right)} \leq 2 \varepsilon$ for $j \geq v$. This proves $\lim _{j \rightarrow \infty}\left\|s_{j}-s\right\|_{S\left(\Lambda_{\mathscr{M}, u, p,\|, \ldots, . .\|}^{2}\right)}=0$ and $s \in S\left(\Lambda_{\mathscr{M}, u, p,\|, \ldots, . .\|}^{2}\right)$ which completes the proof. 
Lemma 5. Let $\mathscr{M}=\left(M_{k}\right)$ be a sequence of Orlicz functions, $p=\left(p_{k}\right)$ be a bounded sequence of positive real numbers and $u=\left(u_{k}\right)$ be a sequence of strictly positive real numbers. If a trigonometric series

$$
\sum_{k=1}^{\infty}\left(a_{k} \cos k t+b_{k} \sin k t\right)
$$

converges strongly for t belonging to a set of positive measure or of second category, then

$$
\lim _{n} \frac{1}{\lambda_{n}} \sum_{k=2}^{n} a_{n k}\left[u_{k} M_{k}\left(\left\|\frac{\lambda_{k-2}\left(a_{k}+b_{k}\right)}{\rho}, z_{1}, \ldots, z_{n-1}\right\|\right)\right]^{p_{k}}=0 .
$$

\section{Competing interests}

The authors declare that they have no competing interests.

\section{Authors' contributions}

All authors have contributed to all parts of the article. All authors read and approved the final manuscript.

\section{References}

[1] A. Alotaibi, M. Mursaleen and S. A. Mohiuddine, Statistical approximation for periodic functions of two variables, J. Funct. Spaces Appl., 2013, Art. ID 491768, 5 pp.

[2] C. Belen and S. A. Mohiuddine, Generalized weighted statistical convergence and application, Applied Mathematics and Computation, 219(18)(2013), 9821-9826.

[3] J. S. Connor, The statistical and strong p-Cesaro convergence of sequences, Analysis, 8 (1988), 47-63.

[4] H. Dutta, Some classes of Cesàro-type difference sequences over n-normed spaces, Adv. Difference Equ., 2013, 2013:286, 13 pp.

[5] A.Esi, Strongly almost summable sequence spaces in 2-normed spaces defined by ideal convergence and an Orlicz function, Stud.Univ.Babes s-Bolyai Math. 27(1) (2012), 75-82.

[6] A.Esi, Strongly lacunary summable double sequence spaces in n-normed spaces defined by ideal convergence and an Orlicz function, Advanced Modeling and Optimization, 14(1)(2012),79-86.

[7] , A. Esi and M. K. Ozdemir, $\Lambda$-strongly summable sequence spaces in n-normed spaces defined by ideal convergence and an Orlicz function, Math. Slavoca,63(4), 829-838.

[8] H. Fast, Sur la convergence statistique, Colloq. Math., 2 (1951), 241-244.

[9] J. A. Fridy, On statistical convergence, Analysis, 5 (1985), 301-313.

[10] J. A. Fridy and C. Orhan, Lacunary statistical convergence, Pacific J. Math., 160 (1993), 43-51.

[11] S. Gähler, Linear 2-normietre Rume, Math. Nachr., 28 (1965), 1-43.

[12] H. Gunawan, On n-Inner Product, n-Norms, and the Cauchy-Schwartz Inequality, Sci. Math. Japonicae, 5 (2001), 47-54.

[13] H. Gunawan and M. Mashadi, On n-normed spaces, Int. J. Math. Math. Sci., 27 (2001), 631-639.

[14] P. Kórus, On $\Lambda^{2}$-strong convergence of numerical sequences revisited, Acta Math. Hungar., 148 (2016), 222-227.

[15] P. Kòrus, On the uniform convergence of double sine integrals with generated monotone coefficients, Period. Math. Hungar., textbf63 (2011), 205-214.

[16] J. Lindenstrauss and L. Tzafriri, An Orlicz sequence spaces, Israel J. Math., 10 (1971), pp.379-390.

[17] L. Maligranda, Orlicz spaces and interpolation, Seminars in Mathematics, 5, Polish Academy of Science, (1989).

[18] A. Misiak, n-Inner product spaces, Math. Nachr., 140 (1989), 299-319.

[19] S. A. Mohiudine and B. Hazarika, Some Classes of Ideal Convergent Sequences and Generalized Difference Matrix Operator, Filomat, 31(6), (2017), 1827-1834. 
[20] M. Mursaleen and A. K. Noman, On some new sequence spaces of non absolute type related to the spaces $l_{p}$ and $l_{\infty}$, Filomat, 25 (2011), 33-51.

[21] J. Musielak, Orlicz spaces and modular spaces, Lecture Notes in Mathematics, 1034, Springer Verlag, (1983).

[22] M. Et, S. A. Mohiuddine and A. Alotaibi, On $\lambda$-statistical convergence and strongly $\lambda$-summable functions of order $a$, J. Inequal. Appl., (2013), 2013:469, 8 pp.

[23] F. Móricz, On $\Lambda$-strong convergence of numerical sequence and Fourier series, Acta Math. Hungar.,54 (1989), $319-327$.

[24] K. Raj, A. Azimhan and K. Ashirbayev, Some generalized difference sequence spaces of ideal convergence and Orlicz functions, J. Comput. Anal. Appl., 22 (2017), 52-63.

[25] K. Raj and S. Pandoh, On certain spaces of double sequences by De la Vallee-Pousin mean, Asian-Eur. J. Math., 8(2015), 1550079, 20 pages.

[26] K. Raj and S. Jamwal, On some generalized statistical convergent sequence spaces, Kuwait J. Sci., 42 (2015), 86-104.

[27] K. Raj and A. Kılıçman, On certain generalized paranormed spaces, Journal of Inequalities and Applications, (2015)(1), $2015: 37$.

[28] K. Raj and A. Kılıçman, On generalized difference Hahn sequence spaces, The Scientific World Journal, , 2014, Article ID 398203 (2014).

[29] T. Šalát, On statistically convergent sequences of real numbers, Math. Slovaca, 30 (1980), 139-150.

[30] E. Savaş and M. Mursaleen, Matrix transformations in some sequence spaces, Istanb. Univ. Fen Fak. Mat. Derg., 52 (1993), 1-5.

[31] I. J. Schoenberg, The integrability of certain functions and related summability methods, American Math. Monthly, 66 (1959), 361-375

[32] B.C.Tripathy and A. Esi, A new type of difference sequence spaces, Int J. Sci. Technol., 11 (2006), 11-14.

[33] A.Wilansky, Summability through Functional Analysis, North-Holland Math. Stud., 85 (1984). 